\title{
Response surface methodology and multivariate analysis of equilibrium headspace concentration of orange beverage emulsion as function of emulsion composition and structure
}

\begin{abstract}
The influence of emulsion composition (i.e. Arabic gum, xanthan gum and, orange oil) and structural emulsion properties (i.e. average droplet size and apparent viscosity) on equilibrium headspace concentration of beverage emulsions was investigated. Increase in average droplet size led to increase the equilibrium headspace concentration of more hydrophilic volatile compounds (i.e. lower $\log$ P) such as ethyl acetate and octanal, but decrease in more hydrophobic volatile compounds such as 3-carene, myrcene and limonene. In most cases, apparent viscosity had significant positive effect on equilibrium headspace concentration. Principle component analysis (PCA) score discriminated the beverage emulsions containing the same orange oil content but different contents of emulsifiers in different classes, thus indicating the significant $(\mathrm{p}<0.05)$ effect of emulsifier fraction on equilibrium headspace concentration. Beverage emulsion containing $22.2 \%(\mathrm{w} / \mathrm{w})$ Arabic gum, $0.52 \%(\mathrm{w} / \mathrm{w})$ xanthan gum and $14.21 \%(\mathrm{w} / \mathrm{w})$ orange oil was estimated to provide the highest equilibrium headspace concentration.
\end{abstract}

Keyword: Arabic gum; Xanthan gum; Orange oil; Equilibrium volatile headspace; Average droplet size; Apparent viscosity; Beverage emulsion 